\title{
Correctness of the Concurrent Approach to Symbolic Verification of Interleaved Models
}

\author{
Felice Balarin \\ Cadence Berkeley Laboratories \\ Berkeley, CA, USA
}

\begin{abstract}
Interleaved models of computations limit the number of system components that can change states simultaneously. This restriction often decreases efficiency of symbolic verification methods based on binary decision diagrams. We propose to ignore this restriction, and allow all components to change states simultaneously. This approach may result in an over-approximation of reachable states. We show that if a system satisfies certain conditions, then the approach computes reachable states exactly. We also show that even if a system does not meet these conditions, it is still possible to compute its reachable states exactly by only partially removing interleaving restrictions. We present experiments that show that the approach improves efficiency of symbolic verification, even when costs of correctness testing are taken into account.
\end{abstract}

\section{Introduction}

Finite-state models can be classified based on rules for deriving behaviors of systems from behaviors of components. Roughly, the models are divided into two classes: interleaved and concurrent. In interleaved models (sometimes also called asynchronous, or disjunctive), a transition of the system corresponds to a transition of a single, or a small number of related components [5, 4, 10, 7]. On the other hand, in concurrent models (sometimes also called synchronous, or conjunctive, or simultaneous), a transition of the system corresponds to transitions of all, or an arbitrary number of components [6, 11, 2]. Traditionally, interleaved models are used for modeling software and asynchronous hardware, while concurrent models are used for synchronous hardware. This distinction is not fundamental: it is quite easy to develop methods for embedding models from one class into another.

The two classes also differ in approaches to their verification. Formal verification tools based on interleaved models typically employ explicit state space search $[4,7]$, while those based on concurrent models often use symbolic search based on binary decision diagrams (BDDs) $[11,2]$. While not fundamental, this distinction has some practical justification. On one side, interleaved models are better for explicit search because they require enumerating enabled transitions, while concurrent models require enumerating all subsets of enabled transitions. On the other side, the requirement that only a single component executes at a time, may cause artificial correlation between components, which may decrease the efficiency of the symbolic search $[3,1]$. 
Motivated by excellent performance of symbolic search methods on concurrent models, there have been several attempts to improve symbolic search for interleaved models. They are either based on extending partial-order methods to symbolic search $[1,8]$, or on changing the order of symbolic search to avoid the artificial-correlation problem [3].

Here, we pursue a different approach, where a system in an interleaved model is analyzed as if its components were composed in a concurrent model. The approach was originally suggested in [3]. It was noted there the approach may produce incorrect results, but rather than exploring the problem further, the authors only remarked that no discrepancies occurred on the set of examples they were considering.

In this paper, we give a characterization of systems for which the concurrent approach is correct. We note that checking exact correctness conditions is hard, and propose several sufficient tests. One of the proposed tests, when it fails, produces a side-result, which may be used to perform correct symbolic search on a model which allows less simultaneous transitions than the concurrent, but still more than the interleaved model. The test is of polynomial complexity, and our experiments indicate that its cost may be more than justified by savings from using the concurrent approach.

In the rest of this paper we first set the context in Section 2. Then, we give the correctness criteria in Section 3, and propose sufficient correctness checks in Section 4. An example is introduced in Section 5, and some experimental results are presented in Section 6. Final comments are given in Section 7.

\section{Preliminaries}

In this section we present an interleaved model of computation. The details of the model are chosen to simplify the presentation of our approach, but the approach is not specific to the presented model. It is quite straightforward to develop similar approaches for other interleaved models of computation.

A system is a finite collection of processes. Each process $P_{i}$ is given by:

- the present state variable $\mathbf{p s}_{i}$ and the next state variable $\mathbf{n s}_{i}$ both ranging over some finite set of local states; we use ps (ns) to denote the vector containing all present (next) state variables, and refer to their valuations as global states,

- the initial states predicate $\mathbf{I}_{i}$ in variable $\mathbf{p s}_{i}$,

- the transition relation predicate $\mathbf{T}_{i}$ in variables $\mathbf{p s}$ and $\mathbf{n s}_{i}$.

Note that $\mathbf{T}_{i}$ can depend on all present state variables in $\mathbf{p s , ~ i . e . ~ t h e ~ p r o c e s s e s ~}$ communicate by observing states of other processes.

An execution of the system is any finite sequence $s_{0}, s_{1}, \ldots, s_{n}$ of global states such that:

1. for all processes $P_{i}: s_{0, i}$ satisfies $\mathbf{I}_{i}$, and

2. for every $j=1, \ldots, n$ there exists a process $P_{i}$ such that $\left(s_{j-1}, s_{j, i}\right)$ satisfies $\mathbf{T}_{i}$ and $s_{j-1, k}=s_{j, k}$ for all $k \neq i$. 
Given a system consisting of processes $P_{i}$, we say that a composition of $P_{i}$ 's is any process that has vector $p s$ of the present state variables of $P_{i}$ 's as its present state variable, vector $\mathbf{n s}$ as its next state variable, and the initial states predicate $\bigwedge_{i} \mathbf{I}_{i}$. In particular, we define the interleaved composition $P_{\|}$to be the composition with the transition relation predicate:

$$
\mathbf{T}_{\|}=\bigvee_{i}\left(\mathbf{T}_{i} \wedge \bigwedge_{j \neq i}\left(\mathbf{p s}_{j}=\mathbf{n s}_{j}\right)\right),
$$

and the concurrent composition $P_{\times}$to be the composition with the transition relation predicate:

$$
\mathbf{T}_{\times}=\bigwedge_{i}\left(\mathbf{T}_{i} \vee\left(\mathbf{p s}_{i}=\mathbf{n s}_{i}\right)\right)
$$

Intuitively, while $\mathbf{T}_{\|}$requires that at every step exactly one process executes, $\mathbf{T}_{\times}$allows any number of processes (including zero) to execute in any step. It is not hard to see that a collection of processes has the same executions as their interleaved composition.

A global state of a system is said to be reachable if it appears in some execution of the system. Computing reachable states is the crucial step in formal verification of safety properties of systems. It is well known that reachable states can be computed by the following fix-point computation:

\section{Algorithm REACH}

1. $\mathbf{R}:=\bigwedge_{i} \mathbf{I}_{i}$

2. $\quad \mathbf{R}:=\mathbf{R} \vee\left[\exists \mathbf{p s}: \mathbf{R} \wedge \mathbf{T}_{\|}\right]_{\mathbf{n s}-\mathbf{p s}}$

until convergence

(We use $[\mathbf{P}]_{\mathbf{x}-\mathbf{y}}$ to denote the predicate obtained by replacing every occurrence of $\mathbf{x}$ in $\mathbf{P}$ with $\mathbf{y}$.)

After $k$ iterations, $\mathbf{R}$ represents exactly those states which appear in some execution of length $k$ or less. This means that even if all $P_{i}$ 's are independent, their present state variables will be correlated in $\mathbf{R}$ (because they must satisfy the interleaving constraint that the sum of number of transitions in individual processes is less or equal to $k$ ). This correlation may cause the BDD representation of $\mathbf{R}$ to grow [3, 1]. In practice, it is often possible to weaken the correlation by computing the fix-point in a somewhat different order, using a partition of $\mathbf{T}_{\| 1}[3]$.

In this paper, we pursue a different approach where $\mathbf{T}_{\|}$is replaced with $\mathbf{T}_{\times}$ in step 2 of algorithm REACH. We refer to this modification of REACH as algorithm CONCUR. Algorithm CONCUR may improve on REACH in several ways: 
- $\mathbf{T}_{\times}$may have a smaller BDD representation than $\mathbf{T}_{\|}((2)$ is certainly simpler than (1), however this does not necessarily translate to a smaller BDD representation),

- algorithm CONCUR may require fewer iterations than REACH,

- intermediate representation $\mathbf{R}$ of reachable states may be simpler in CONCUR because the interleaving constraint is removed.

Of course, these are all heuristics, and it is possible to construct examples where REACH is more efficient than CONCUR, but we will show in Section 6 that these heuristics are supported by experiments.

\section{Correctness of the Concurrent Approach}

Algorithm CONCUR may not compute reachable states correctly. In this section, we will first show that it always computes a superset of reachable states, and then establish some sufficient conditions, such that if a system satisfies them, then CONCUR computes reachable states exactly. Finally, we will show that in case these conditions are not met, it is still possible to construct a composition that is more concurrent than $P_{\|}$(thus retaining some benefits of $P_{\times}$), but has the same reachable states as $P_{\|}$.

Proposition 1. Let $P^{\prime}$ be some composition of $P_{i}$ 's, and let $\mathbf{T}^{\prime}$ be its transition relation predicate. If $\mathbf{T}_{\|} \Rightarrow \mathbf{T}^{\prime}$ is valid, then every execution of $P_{\|}$is also an execution of $P^{\prime}$.

The proof is straightforward from the definition of an execution.

It is easy to check that $\mathbf{T}_{\|} \Rightarrow \mathbf{T}_{\times}$is valid. Thus, the following result holds by Proposition 1.

Corollary 2. Every state reachable in $P_{\|}$is also reachable in $P_{\times}$.

Before we examine when the opposite holds, we need some definitions. We call a pair of global states a global transition. A global transition that satisfies the transition relation of some process $P$ is said to be enabled in $P$. Given a transition $(s, q)$, its set of active processes is defined by:

$$
\left\{P_{i} \mid s_{i} \neq q_{i}\right\} .
$$

We say that a sequence $s_{0}, \ldots, s_{N}$ of global states is a serialization of some global transition $(s, q)$ if:

- $s_{0}=s, s_{N}=q, N$ is the number of active processes of $(s, q)$, and

- there exists an ordering $P_{i_{1}}, \ldots, P_{i_{N}}$ of active processes of $(s, q)$ such that for every $j=1, \ldots, N$ :

- $\left(s_{j-1}, s_{j, i_{j}}\right)$ satisfies $\mathbf{T}_{i_{j}}$, and

- for all $k \neq i_{j}: s_{j-1, k}=s_{j, k}$. 
A composition of $P_{i}$ 's is serializable iff every enabled transition in every one of its reachable states has a serialization.

Proposition 3. If a composition $P^{\prime}$ of $P_{i}$ 's is serializable, then every state reachable in $P^{\prime}$ is also reachable in $P_{\|}$.

Indeed, if a state $s$ is reachable in $P^{\prime}$ it must appear in some execution $r^{\prime}$ of $P^{\prime}$. From $r^{\prime}$, we can construct an execution $r$ of $P_{\|}$, by (i) replacing every transition in $r^{\prime}$ with its serialization, and (ii) eliminating any adjacent occurrences of the same state. Obviously, $s$ also appears in $r$, thus it is reachable in $P_{\|}$.

Corollary 4. If $P_{\times}$is serializable, then it has the same reachable states as $P_{\|}$.

If $P_{\times}$is not serializable, algorithm CONCUR can be used to compute an over-approximation of reachable states. Or, the concurrent approach may be modified, as indicated by the following result.

Proposition 5. If $P^{\prime}$ is a composition of $P_{i}$ 's such that:

- it is serializable,

- its transition relation predicate has the form $\mathbf{T}_{\times} \wedge \neg \mathbf{C}$, where $\mathbf{C}$ is such that any global transition satisfying $\mathrm{C}$ has at least two active processes,

then, $P^{\prime}$ has the same reachable states as $P_{\|}$.

By Proposition 3 states reachable in $P^{\prime}$ are also reachable in $P_{\|}$, because $P^{\prime}$ is serializable. To prove the opposite, observe that any global transition satisfying $\mathbf{T}_{\|}$has at most one active process. It follows that $\mathbf{T}_{\|} \wedge \mathbf{C}$ is not satisfiable. From this fact and the validity of $\mathbf{T}_{\|} \Rightarrow \mathbf{T}_{\times}$, we have that $\mathbf{T}_{\|} \Rightarrow\left(\mathbf{T}_{\times} \wedge \neg \mathbf{C}\right)$ is valid, and the desired result is immediate from Proposition 1.

In the following section we will propose a conservative serializability test, which as a side-product computes $\mathbf{C}$ satisfying the conditions of Proposition 5.

\section{Checking Serializability}

Deciding whether a process is serializable is a hard problem in general. It can be shown that just checking whether a single global transition has a serialization, is NP-complete. Fortunately, we rarely have to solve the general problem. It is possible that some modeling style generate only serializable systems. An example of such a style is the model of asynchronous circuits presented in [5]. It is also well known that any system can be made serializable by refining the granularity of its transitions [10]. However, this may unacceptably increase its size.

Often, it is possible to define simple syntactic sufficient serializability tests. For example, if in our model of computation local state spaces are decomposed into observable and hidden components, and any transition that changes observable states depends only on the local state, then $P_{x}$ is serializable. This check can be performed locally on $\mathbf{T}_{i}$ 's. It is similar to a single-reference rule in [10]. 
Serializability can also be established by analyzing the dependency graph of the system, in which nodes correspond to processes, and there is an edge $(i, j)$ whenever $\mathbf{T}_{j}$ depends on $\mathbf{p s}_{i}$. If the graph of the system is acyclic, then the system is serializable. In this case, we may use a fixed serialization order, such that a process is always executed after all the processes that depend on it.

\subsection{A test based on disabling}

Next, we will present a slightly more complex sufficient serializability test. The complexity of this test is quadratic in the number of processes. If a system is not serializable, the results of the test can be used to modify $P_{\times}$as in Proposition 5.

The key concept in the test is transitions disabling one another. First, we formally define a local transition of some process $P_{i}$ as a predicate of the form $\mathbf{p s}_{i}=s \wedge \mathbf{n s}_{i}=q$, where $s$ and $q$ are two distinct local states of $P_{i}$.

We say that a local transition $\mathbf{t}$ may disable some other local transition $\mathbf{u}$ in some other process, if there exists a global state in which they are both enabled, but $\mathbf{u}$ is no longer enabled if (only) $\mathbf{t}$ is executed. Formally, the disable relation $D s b l$ contains all pairs $(\mathbf{t}, \mathbf{u})$ such that $\mathbf{t}$ is a local transition of some process $P_{i}$, $\mathbf{u}$ is a local transition of some different process $P_{j}$, and

$$
\mathbf{T}_{i} \wedge \mathbf{T}_{j} \wedge \neg\left[\mathbf{T}_{j}\right] \mathbf{p s}_{z} \leftarrow \mathbf{n s} \mathbf{s}_{i} \wedge \mathbf{t} \wedge \mathbf{u}
$$

is satisfiable.

Proposition 6. If the disable relation is acyclic, then $P_{\times}$is serializable.

If the disable relation is acyclic, then it induces a partial order on active process of some transition. To serialize that transition, we may choose any total ordering of active processes that is consistent with the inverse of that partial order. In other words, given a set active processes, we always choose to execute one that may not disable any other still active process. Since the disable relation is assumed to be acyclic, such a choice is always possible.

Proposition 7. If Cut $\subseteq D$ sbl is such that Dsbl-Cut is acyclic, then the composition of $P_{i}$ 's with the transition relation predicate:

$$
\mathbf{T}_{\times} \wedge \neg \bigvee_{(\mathbf{t}, \mathbf{u}) \in C u t} \mathbf{t} \wedge \mathbf{u}
$$

has the same reachable states as $P_{\|}$.

This result follows from Proposition 5. To apply this proposition, we need to show that:

- any global transition satisfying $\bigvee \mathbf{t} \wedge \mathbf{u}$ has at least two active processes, $(\mathbf{t}, \mathbf{u}) \in C u t$

- the composition of $P_{i}$ 's with the transition relation (3) is serializable. 
The former is straightforward, and to show the latter observe that any set of local transitions that form a cycle in $D s b l$ must contain at least one pair of transitions in Cut, but (3) ensures that no pair of transitions in $C u t$ may appear in the same global transition.

The size of $D s b l$ is at worst quadratic in the total number of local transitions, which is obviously linear in the number of processes. Checking whether $D s b l$ is acyclic can be done in linear time, by a simple traversal of its graph. The same traversal may be used to generate the $C u t$ set. It is a reasonable heuristic to keep Cut small, but finding the smallest $C u t$ is not practical, because it is known to be NP-complete.

In case the number of local transitions is much larger than the number of processes, it may be more efficient to find the cut predicate $\mathbf{C}$ using the following modification of the Floyd-Warshall algorithm [9], which avoids explicit enumeration of local transitions:

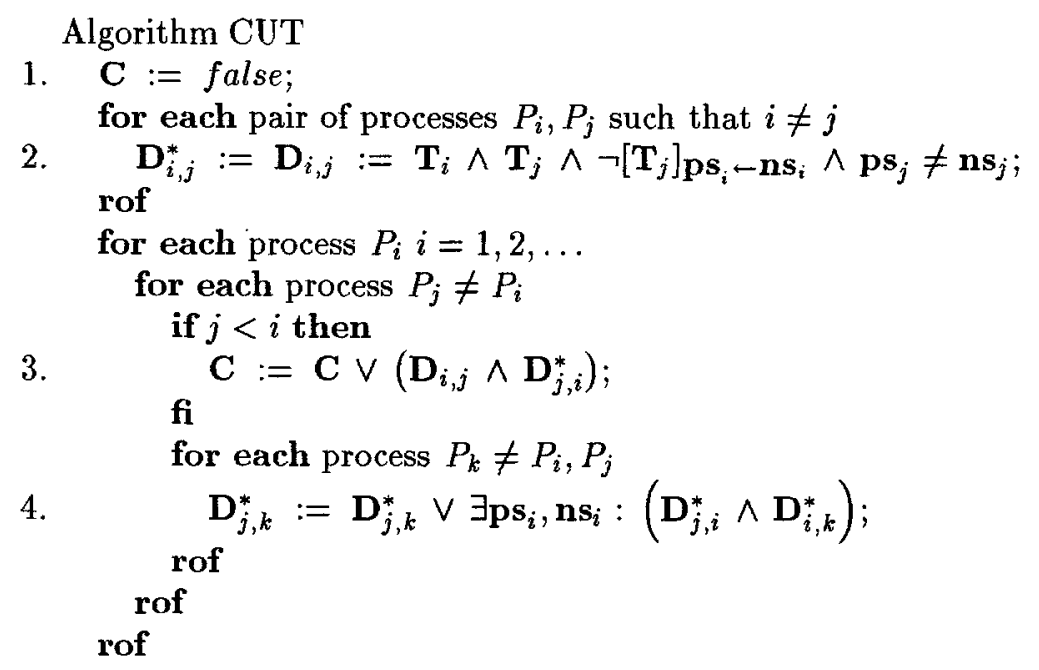

Intuitively, predicate $\mathbf{D}_{i, j}$ characterizes the portion of $D s b l$ corresponding to processes $P_{i}$ and $P_{j}$, and $\mathbf{D}_{i, j}^{*}$ eventually characterizes its transitive closure. After the $i$-th pass through the main loop, a pair of local transitions in $P_{j}$ and $P_{k}$ satisfies $\mathbf{D}_{j, k}^{*}$ iff there exists a path between them in $D s b l$ that does not visit any process with index higher than $i$.

At the end, the cut predicate $\mathbf{C}$ characterizes at most one pair of local transitions per each independent cycle in $D s b l$. Of course, it is not necessarily the smallest such set.

There may be some cycles in $D s b l$ that are not cut by $\mathbf{C}$, but any such cycle must include two local transitions of the same process. Since transitions of the same process cannot occur simultaneously even in $\mathbf{T}_{\times}$, it follows that $\mathbf{T}_{\times} \wedge \neg \mathbf{C}$ still satisfies conditions of Proposition 5.

There is an interesting relation between the proposed algorithm and partialorder reduction methods such as [13]. These methods attempt to accelerate state 
space search by not executing all enabled transitions in a state. Instead, the socalled ample set of transition is chosen to be executed, such that every transition not in the ample set is independent of all the transitions in it. Our approach has similar objectives, except that it attempts to execute in one step as many transitions as possible, rather than trying to execute only a subset of transitions. Since the cut predicate $\mathbf{C}$ prevents only a subset of transitions in $D s b l$ from executing concurrently, and since all the pairs of transitions in $D s b l$ are dependent, it follows that whenever a partial order method can execute only one transition from a pair, our algorithm will execute both of them in one step.

The disabling based test is less conservative than the test based on decomposing the state space into observable and hidden components. Note that hidden transition can never disable any other transitions (because they are not observable). Also note that observable transitions can never be disabled (because we assume they depend only on the local state). These two observations imply that the disable relation is acyclic.

The disabling based test is also less conservative than the test based on the dependency graph. In fact, the dependency graph may be seen as an abstraction of the disable relation where all transitions of the same process are merged into a single node.

\section{An Example}

Consider the system shown in Figure 1. A typical process $P_{i}$ starts in the initial state 0 , moves to 1 if its left neighbor is in state 0 , then moves to 2 if its right neighbor is in state 1 , and finally returns to the initial state to repeat this behavior indefinitely. Boundary processes $P_{1}$ and $P_{N}$ are similar, except that their state changes are unconditional, if their neighbors do not exist.

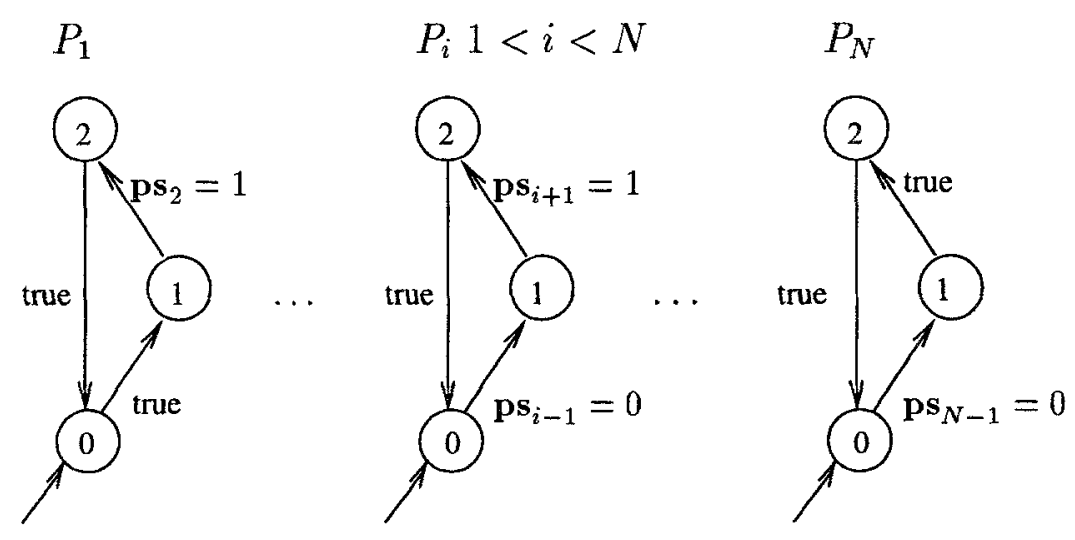

Fig. 1. A system with $N$ processes.

The example in Figure 1 is constructed to highlight circumstances in which 
the concurrent symbolic verification approach out-performs other approaches. First, observe that partial order methods are of little use in this example because most of the transitions are dependent. For comparison to other symbolic approaches, it is useful to analyze how the system can reach the state in which all processes are in state 2 . That state is interesting because it is hard to reach, in the sense that the REACH algorithm reaches it later than any other state. The shortest path to that state requires $2 N$ process executions in the following order:

$$
P_{1}, P_{2}, \ldots, P_{N}, P_{N}, P_{N-1}, \ldots, P_{1} .
$$

Thus, the REACH algorithm requires $2 N+1$ iterations (the last one to ensure that no new states are added). Approaches based on partitioning the transition relation can reduce this number, but since they typically process individual transition relations in a fixed order, at least one half of the sequence above will be in the wrong order, and they will still require $O(N)$ iterations.

On the other hand, the CONCUR requires only 3 iterations, since state $(2, \ldots, 2)$ can be reached in two steps, first by executing concurrently all processes to reach state $(1, \ldots, 1)$, and then executing all of them once more to reach $(2, \ldots, 2)$.

It is not hard to see that the $D s b l$ relation is acyclic in this case. For example, the graph of the $D s b l$ relation for the system with 4 processes is shown in Figure 2. However, if $P_{1}$ is modified so that it can move from state 0 only if $P_{N}$ is in state 0 , the $D s b l$ relation would have a cycle containing $0 \rightarrow 1$ transitions of all the processes. The CONCUR would now compute a strict superset of reachable states. For example, it would indicate that state $(1, \ldots, 1)$ is reachable, even though in every truly reachable state at least one process must be in state 0 . To fix this problem it suffices to choose $C u t=\left\{\left(\mathbf{p s}_{1}=0 \wedge \mathbf{n s}_{1}=1, \mathbf{p s}_{2}=0 \wedge \mathbf{n s}_{2}=\right.\right.$ $1)\}$ and to modify $\mathbf{T}_{\times}$as in (3). This would prevent $0 \rightarrow 1$ transitions in $P_{1}$ and $P_{2}$ from occurring simultaneously. With this modification, CONCUR computes reachable states correctly. The number of iterations is increased, but it is still a constant independent of $N$.

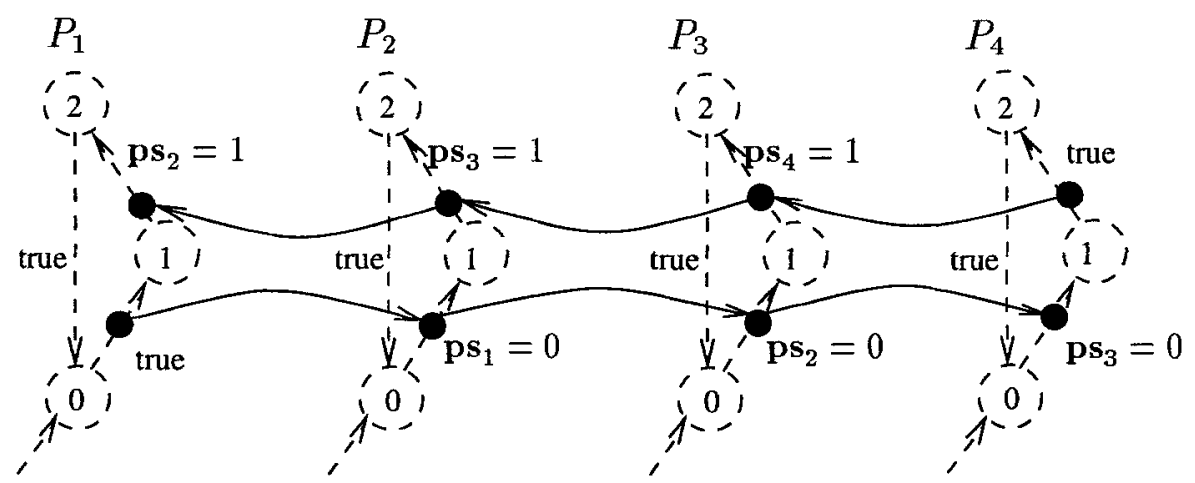

Fig. 2. The graph of the disable relation. 


\section{Experiments}

We have implemented algorithms REACH, CUT, and a modification of algorithm CONCUR where $\mathbf{T}_{\times}$has been modified as in Proposition 5, using the cut predicate $\mathbf{C}$ computed by CUT. We have named this algorithm CON_CUT. Of course, if the disable relation is acyclic, CUT will compute $\mathbf{C}=$ false, and CON_CUT becomes CONCUR. For comparison purposes, we have also implemented an algorithm we call PART, which is an exact reachability algorithm based on partitioning $\mathbf{T}_{\|}$, similar to those discussed in [3]. All algorithms are implemented on top of the same BDD package [12]. We note that the partitioning approach could also apply to CON_CU'T, but we do not have any experimental results for that combination.

We have applied these algorithms to two groups of examples: a cyclic version of the example described in Section 5 , and an asynchronous tree arbiter described in [5]. The latter arbitrates access to a shared resource among $2^{n}$ processors. It is configured as a binary tree of $2^{n}-1$ cells, each arbitrating between two processors, or two cells at a lower level of the tree.

Table 1. Results for the example from Section 5.

\begin{tabular}{c|r|r|r|r|r}
\multirow{2}{*}{ alg. } & \multicolumn{5}{|c}{$\#$ of processes } \\
\cline { 2 - 6 } & 10 & 20 & 30 & 40 & 50 \\
\hline CUT & $1.3 \mathrm{~s}$ & $10 \mathrm{~s}$ & $34 \mathrm{~s}$ & $82 \mathrm{~s}$ & $160 \mathrm{~s}$ \\
CON_CUT & $.03 \mathrm{~s}$ & $.07 \mathrm{~s}$ & $.11 \mathrm{~s}$ & $.16 \mathrm{~s}$ & $.23 \mathrm{~s}$ \\
\hline REACH & $.23 \mathrm{~s}$ & $1.6 \mathrm{~s}$ & $7.9 \mathrm{~s}$ & $17 \mathrm{~s}$ & $98 \mathrm{~s}$ \\
\hline PART & $.48 \mathrm{~s}$ & $6.5 \mathrm{~s}$ & $50 \mathrm{~s}$ & $225 \mathrm{~s}$ & $719 \mathrm{~s}$
\end{tabular}

Execution times for the example in Section 5 are summarized in Table 1. As expected, the concurrent approach significantly outperforms other approaches. Furthermore, CON_CUT requires only seven iterations, regardless of the number of processes. Also, intermediate sets of reachable states generated by CON_CUT had smaller BDDs than those generated by REACH or PART. In this case, CU'T is not an efficient way to check serializability. In fact, it is less expensive to compute reachable states with $\mathrm{REACH}$ than to compute the cut predicate C with CUT. However, with only three transition per process, and the disable relation that grows linearly with the number of processes, this example is well suited for explicit traversal of the disable relation.

Execution times for the tree arbiter are shown in Table 2. Again, CON_CUT outperforms REACH and PART. In this case, the disable relation is acyclic, so CUT serves only as a proof of correctness, but it is not required for computing reachable states. Note that for 16 or more processors combined run times of CU'T and CON_CUT are shorter than rum times of REACH or PART. Furthermore, the run time of CUT grows slower than run times of other algorithms. 
Table 2. Results for the asynchronous tree arbiter.

\begin{tabular}{c|r|r|r|r}
\multirow{2}{*}{ alg. } & \multicolumn{3}{|c}{ \# of processors } \\
\cline { 2 - 5 } & 4 & 8 & 16 & 32 \\
\hline CUT & $.34 \mathrm{~s}$ & $2.7 \mathrm{~s}$ & $21 \mathrm{~s}$ & $172 \mathrm{~s}$ \\
CON_CUT & $.05 \mathrm{~s}$ & $.41 \mathrm{~s}$ & $9.9 \mathrm{~s}$ & $695 \mathrm{~s}$ \\
\hline REACH & $.09 \mathrm{~s}$ & $2 \mathrm{~s}$ & $391 \mathrm{~s}$ & $>5 \mathrm{~h}$ \\
\hline PART & $.14 \mathrm{~s}$ & $1.9 \mathrm{~s}$ & $81 \mathrm{~s}$ & $1 \mathrm{~h}$
\end{tabular}

\section{Conclusions and Future Work}

We have shown that relaxing interleaving requirements may improve efficiency of symbolic verification, but it may also lead to incorrect (too conservative, to be precise) verification results. We have proposed several correctness tests, and showed that results of one of these can be used to only partially relax interleaving requirements in a manner that guarantees correctness.

All proposed tests try to establish a priori that the concurrent approach is correct for the system at hand. There is also an alternative where one applies the concurrent approach without any checks. If the computed set of reachable states contains no unsafe states, the system is declared correct. If that is not the case, the verification tool produces a path from some of the initial states to some of the unsafe states. It that path contains only serializable transitions, the system is declared incorrect. Otherwise, the transitions in the path that are not serializable are eliminated from $\mathbf{T}_{\times}$as in (3), and the procedure is repeated.

It is not hard to show that the described procedure terminates in a finite number of iterations. However, the provable bound is of little practical use, because the procedure will be competitive to other approaches only if the number of iterations is much smaller than in the worst case.

Results of this paper are applicable to verification of safety properties, but the approach could (and should in the future) be extended to liveness properties as well. The future work should also include more experiments to better understand when the proposed approach is beneficial.

\section{References}

1. Rajeev Alur, R. K. BraytonT. A. Henzinger, S. Qadeer, et al. Partial-order reduction in symbolic state space exploration. In O. Grumberg, editor, Proceedings of Computer Aided Verification: 9th International Conference, CAV'97, Haifa, Israel, 22-25 June. Springer-Verlag, 1997. LNCS vol. 1254.

2. R.K. Brayton, A. Sangiovanni-Vincentelli, G.D. Hachtel, F. Somenzi, A. Aziz, S.T. Cheng, S. Edwards, S. Khatri, Y. Kukimoto, S. Qadeer, R.K. Ranjan, T.R. Shiple, G. Swamy, T. Villa, A. Pardo, and S. Sarwary. VIS: A system for verification and synthesis. In Rajeev Alur and Thomas A. Henzinger, editors, Proceedings of Computer Aided Verification: 8th International Conference, CAV'96, Rutgers, NJ, July, 1996, Springer-Verlag, 1996. LNCS vol. 1102. 
3. J. R. Burch, Edmund M. Clarke, David E. Long, Ken L. McMillan, and David L. Dill. Symbolic model checking for sequential circuit verification. IEEE Transactions on Computer-Aided Design of Integrated Circuits and Systems, 13(4):401-24, April 1994.

4. D. L. Dill, A. J. Drexler, A. J. Hu, and C. H. Yang. Protocol Verification as a Hardware Design Aid. In Proceedings of ICCD, pages 522-525, October 1992.

5. D.L. Dill. Trace Theory for Automatic Hierarchical Verification of SpeedIndependent Circuits. The MIT Press, Cambridge, Mass., 1988. An ACM Distinguished Dissertation 1988.

6. Z. Har'El and R. P. Kurshan. Software for analysis of coordination. In Proceedings of the International Conference on System Science, pages 382-385, 1988.

7. Gerard J. Holzmann. Design and validation of computer protocols. Englewood Cliffs, N.J. : Prentice Hall, 1991.

8. R. P. Kurshan, V. Levin, M. Minea, D. Peled, et al. Verifying hardware in its software context. In Digest of Technical Papers of the 1997 IEEE International Conference on CAD, pages 742-9, November 1997.

9. Eugene L. Lawler. Combinatorial optimization : networks and matroids. Holt, Rinehart and Winston, 1976.

10. Zohar Manna and Anir Pnueli. Verification of concurrent programs: The temporal framework. In R. Boyer and J. Moore, editors, Correctness Problem in Computer Science, pages 215-273. Academic Press, 1981.

11. Kenneth L. McMillan. Symbolic Model Checking. Kluwer Academic Publishers, 1993.

12. F. Somenzi. CUDD : CU Decision Diagram Package, June 1996. User's Manual.

13. P. Wolper and P. Godefroid. Partial-order methods for temporal verification. In E. Best, editor, Proceedings of CONCUR '93. 4th International Conference on Concurrency Theory, Hildesheim, Germany, 23-26 Aug. Springer-Verlag, 1993. 